\title{
Reductive Mastoplasty in Gigantomastia with Inferior Pedicle Flap and Free Graft of the Areola-Nipple Complex
}

\section{Tamayo Carbon $\mathrm{AM}^{1}$, Vila Garcia $\mathrm{E}^{2 *}$, Orozco Jaramillo $\mathrm{MA}^{3}$, Hurtado Vidal $J^{4}$, Cedillo Lopez RM ${ }^{5}$ and Cairos Baez JN ${ }^{6}$}

${ }^{1}$ Specialist of II degree in Plastic Surgery and Caumatology, Surgical Hospital Hermanos Ameijeiras, Cuba

${ }^{2}$ Specialist I degree in General Surgery, Surgical Hospital Hermanos Ameijeiras, Cuba

${ }^{3} 3$ rd year Resident of Plastic Surgery and Caumatology, Surgical Hospital Hermanos Ameijeiras,

Cuba

${ }^{4}$ Specialist of I degree in Plastic Surgery and Caumatology, Surgical Hospital Hermanos Ameijeiras, Cuba

${ }^{5} 4^{\text {th }}$ year Resident of Plastic Surgery and Caumatology, Surgical Hospital Hermanos Ameijeiras, Cuba

${ }^{6}$ Specialist of II degree in Plastic Surgery and Caumatology, Surgical Hospital Hermanos Ameijeiras, Cuba

*Corresponding author: Eyleen Vila Garcia, Assistant Professor, Services of Plastic Surgery and Caumatology and General Surgery, Surgical Hospital Hermanos Ameijeiras, Havana City, Cuba, Tel: 53232327; Email: hielenvg@infomed.sld.cu

\section{Abstract}

Introduction: Gigantomastia is the greatest expression of breast hypertrophy and produces great biopsychosocial suffering for patients. Its treatment consists of reductive mastoplasty through combinations of surgical techniques.

Objectives: To evaluate the results of the surgical treatment of gigantomastia with the reductive mastoplasty technique with a lower pedicle flaps and frees grafting of the nipple areola complex.

Method: A descriptive, prospective and longitudinal study was carried out with 50 patients with a diagnosis of gigantomastia who were treated at the breast dysmorphia consultation of the Plastic Surgery service of the Hermanos Ameijeiras Hospital in the period from January 2016 to December 2019. All they were treated with the inferior pedicle surgical technique and free grafting of the areola-nipple complex.

Results: The average age was 41.4 years, with a standard deviation of \pm 12.7 years. The preoperative volume of each breast ranged between 1500 and 4500 gr. It was dried between 700 and 3500 gr. of breast tissue, obtaining an average postoperative volume of $706.80 \mathrm{gr}$., with extreme values of 1000 and $500 \mathrm{gr}$. The late complications were the most frequent in 10 patients with graft hypopigmentation. The results obtained were good in 46 patients and all were satisfied.

Conclusions: Reductive mastoplasty with lower pedicle flap and free graft of the areola-nipple complex is a surgical treatment option for gigantomastia that offers good results, few complications and high satisfaction for patients.

Keywords: Gigantomastia; Reductive mastoplasty

\section{Introduction}

The breast is an anatomical region that plays a predominant role in the cultures, art, philosophy, and religion of different peoples and ethnic groups. Its presence consolidates the feminine personality, has a high sexual meaning and is directly related to human reproduction. Its shape and volume is diverse and its morphology varies 


\section{International Journal of Transplantation \& Plastic Surgery}

during the woman's life. Frequent weight changes, pregnancy, breastfeeding, hormonal imbalances (prolactin, estrogens and progestagens), as well as alterations related to glandular tissue and skin, bring aesthetic and functional disorders in them, with physical and psychological repercussions in women [1-3].

Breast hypertrophy, macromastia or gigantomastia, is a medical condition of the connective tissues of the breast characterized by an abnormal increase in the volume of the breast. It can vary from mild to gigantomastia in cases where the breast volume exceeds 1500 to 2500 gr. It was first described by Palmuth in 1648 as benign progressive growth, which can occur in one or both breasts. Dafydd defined it as excess breast weight of approximately $3 \%$ of total body weight. Chiu et al. from the University of Cambridge in New York differentiate between macromastia and gigantomastia, in that in macromastia the excess tissue is less than $2.5 \mathrm{~kg}$ and in gigantomastia it is greater [4-7].

Breast hypertrophy is a pathology that requires surgical treatment. Abnormal increase in breast volume is commonly a cause of physical and emotional distress in women. The symptoms vary from local symptoms such as infections and dermatitis; even regional ones such as cervical pain, back pain, low back pain, chest deformity, pain and dermatological injuries to the shoulders from the use of the adjuster, pulmonary emphysema, symptoms associated with compression of the ulnar nerve and psychological symptoms. Sharma believes that hypertrophy of the breast tissue can affect both breasts equally, but usually affects one more than the other, thus conditioning breast asymmetry [8-10].

The breast reduction of women suffering from gigantomastia significantly favors their quality of life. In plastic surgery the procedure to reduce the size of the breasts is called reducing mammoplasty, reducing mastoplasty or reduction mammoplasty. This operation consists of reducing the fat, breast tissue and skin of the breasts in order to improve the shape. In addition, it allows them to be kept lighter, smaller and firmer, preserves the viability of the Areola-Nipple Complex (NAC), and ensures functional sensitivity and capacity for lactation. You can also reduce the size of the areola and nipple. Reductive mammoplasty is one of the most representative examples of the interface between reconstructive surgery and aesthetics. Reductive free graft NAC mastoplasty is indicated in any circumstance in which conventional flap reduction mastoplasty techniques put the vascularization of the NAC at risk, such as patients who have symptoms of breast enlargement, breast asymmetry, or who require reconstruction posmastectomy in order to equalize the breast against lateral and achieve symmetry. That is why this procedure is considered within the group of functional or reconstructive treatment techniques [11].
Villandre and Thorek in 1911 carried out the first free graft reduction of the NAC. Biesenberger describes an extensive glandular resection. Schwarzman began the era of de-epithelization in 1930 by basing the circulation of the areola and nipple on a dermal flap; this fundamental principle has allowed the formation of pedicled flaps. Maliniac in 1953 proposed the resection of most of the mammary gland and its reconstruction with a free graft of the NAC and a lower flap of preserved breast tissue. Wise in 1956 contributes a model for designing the lateral flaps and resection zones using a padlockshaped pattern introduced by Neckoff in 1938, which takes the Cordelia bra models and results in an inverted "T" scar. The modern history of reductive mammoplasty begins with Strombeck in 1960, which modifies Wise's mold and creates a horizontal bipedicled flap technique. The most recent techniques are based on old publications with additional surgical details that seek to improve the resulting scar, which should be as small as possible. This motivated surgeons to seek modifications to the traditional " $\mathrm{T}$ " technique, resulting in "J" shapes (Regnault 1974) that are applied according to the surgeon's convenience and abilities. In 1977 Courtis and Goldwyn, using the Mckissock cast, designed a lower base flap by removing skin and dermoglandular tissue from the new site of the areola with a conventional " $\mathrm{T}$ " closure. Robbins in 1977 designed a pyramidal flap with a narrow lower base and a "T" closure, being currently preferred in plastic surgery services in various American and European countries. It transposes the NAC into a lower base pyramidal dermoglandular pedicle, thus preserving its viability and sensitivity [12-16].

There are situations in which reducing breast volume with scar sparing pediculated techniques can put the survival of the NAC at risk. This justifies the validity of the surgical procedures that reduce large breast volumes at the expense of the graft itself and the motivation to evaluate the results of the surgical treatment of gigantomastia with the reductive mastoplasty technique with lower pedicle and NAC graft.

\section{Method}

A prospective, descriptive, and longitudinal study was conducted that included 50 patients who attended the breast dysmorphia consultation of the Plastic Surgery service of the "Hermanos Ameijeiras" Hospital in the period from January 2016 to December 2019. Sample consisted of female patients between 19 and 70 years of age, who were diagnosed with gigantomastia, had no acute dermatological pathology in the breasts or local infection, and had satisfied parity. Patients with decompensated acute or chronic diseases, smokers, and psychiatric disorders were excluded.

First Consultation: Preoperative and pre-anesthetic checkup indicated. In the case of patients with an oncological history, 


\section{International Journal of Transplantation \& Plastic Surgery}

it is requested to be suitable for surgery by treating specialty, as well as the suspension of medications that interfere with coagulation or prolong bleeding times (non-steroidal antiinflammatory drugs, aspirin, ibuprofen, vitamin E, Omegas 3-6-9, anticoagulants). The wishes and expectations of each patient are determined. Different surgical options, benefits and possible complications are explained to them. Doubts are clarified, the informed consent is signed and hospital admission is indicated once the patient is fit for surgery.

Preoperative Indications: Enter the day before surgery, normal diet is indicated until 6:00 pm and liquid until 10:00 $\mathrm{pm}$, to start fasting from $12: 00 \mathrm{pm}$, antibiotic prophylaxis and diazepam $5 \mathrm{mg}$ orally 10:00 pm.

Preoperative Breast Volume Estimation: It was obtained by means of the volume distribution method according to Bouman (observing the Archimedean principle). It consists of immersing the breast in a container with water and the volume of displaced water determines the breast volume in grams when measuring with a container graduated in milliliters, where $1 \mathrm{cc}$ equals 1 gr [17].

Preoperative Planning and Surgical Technique: Front profile and oblique photographic record is made. In a standing position, with the arms at the sides of the body, the submammary groove is marked up to the anterior axillary line, a vertical line is drawn from the midpoint of the clavicle that, passing through the nipple, reaches the submammary groove, dividing the breast in two halves, internal and external. The new position of the NAC is located; a circle with a diameter of 4.5 to $5 \mathrm{~cm}$ is marked on said point. The tissue to be resected is planned with an inverted T-closure in such a way that the resulting distance from the neo NAC to the submammary fold is 5 to $7 \mathrm{~cm}$ (Figure 1).

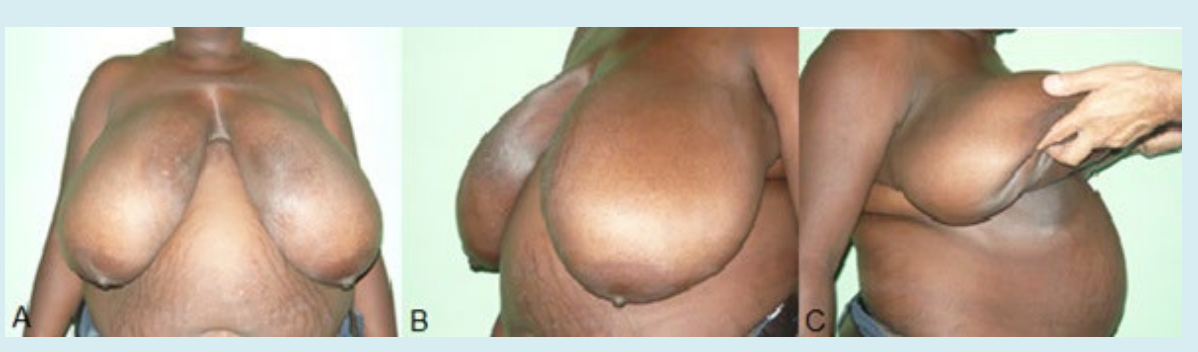

Figure 1: Gigantomastia plus severe ptosis. A: Front view. B: Oblique view. C: Cutaneous changes in segment four of the breast in profile.

Surgical Technique: General orotracheal anesthesia, patient in supine position with arms abducted and shoulders in a horizontal position, asepsis and antisepsis with iodine alcohol, sterile field cloths, incision is made with a \# 15 scalpel to demarcate the previously planned surgical area, performs exeresis of the NAC as a free graft of total skin, which is kept protected with compresses moistened in saline. According to the Schwartzman maneuver, the lower base pedicle flap is de-epithelialized and dissected with a \# 22 scalpel or an electric scalpel up to the pectoral fascia. Excess dermoglandular tissue is removed and sent to pathology for study. The inferior pedicle flap is fixed to the pectoral fascia with a 3-0 non-absorbable suture, creating contention and projection of the breast, preventing its future flattening. The lateral pedicles are sutured, closure is continued by planes until the skin ending with an inverted " $T$ " closure with a nonabsorbable 2-0 or 3-0 suture, after rigorous homeostasis and placement of suction drains. The NAC is grafted using Brown fixation, sterile dressings are placed and the breasts are immobilized with an elastic bandage without exerting pressure.

Posoperative Indications: Relative rest, vital signs, hydration, analgesics and start orally at 6 hours with a liquid diet according to tolerance. It is valued to remove drains after 24 hours according to content $(<10 \mathrm{ml})$. Cures dry within 24 hours. Assess the state of the wound and coping with the edges, as well as volumetric symmetry.

Posoperative Evolution: The general state of the wounds, appearance, coloration and coping of the edges are evaluated every 24 hours up to 3 days. If there were no complications and drains are removed, the patient is discharged with weekly follow-up until completing the first month. Then it is consulted every 3 months until the year. The graft is discovered after 5 days, the removal of stitches from the areola after 10 days and between 18 to 21 days the others are evaluated. We recommend wearing a bra continuously for the first 3 months. A photographic study of the forehead, profile and oblique is carried out in the immediate posoperative period, then one month, 6 months and one year (Figure 2). 


\section{International Journal of Transplantation \& Plastic Surgery}

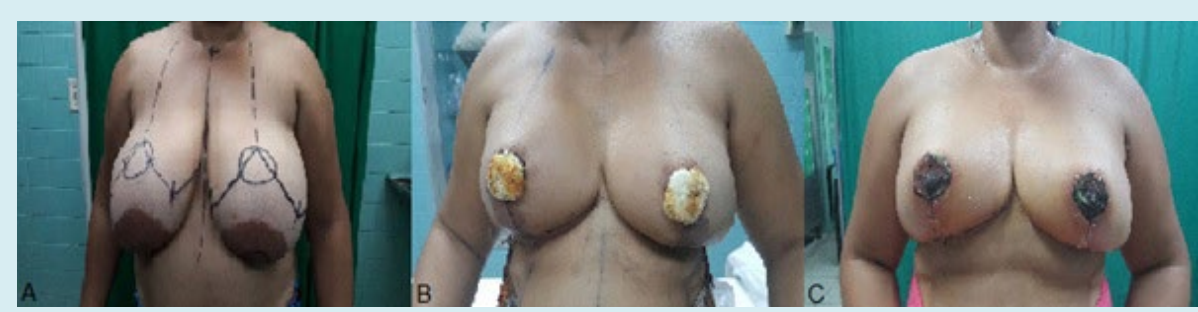

Figure 2: Gigantomastia plus severe ptosis - front view. A: Preoperative- planning of the surgical area. B: Immediate posoperative. C: Posoperative after 21 days.

Diagnosis of Gigantomastia: Based on the Lalardrie and Jouglard classification of 1973 , which states that when the breast volume in $\mathrm{cm}^{3}$ exceeds $50 \%$ of "normal", there is some degree of breast hypertrophy and it is considered gigantomastia at breast volumes of $1500 \mathrm{ml}$ or plus. The amount of resected tissue was calculated by the weight of the piece of breast tissue on an electronic scale graded in grams [18].

Evaluation of Aesthetic Results: They were classified as good, fair and bad taking into account the following criteria:

- Good: NAC in a suitable position, symmetry in the shape and position of the breasts, disappearance of symptoms, absence of complications and inconspicuous scar.

- Regular: Three of the above criteria are met.

- Bad: Less than three criteria are met.

Patient satisfaction described as satisfied and unsatisfied was also assessed.

Analysis and Processing of Information: The information was recorded in data collection sheets and later in a database made in the Microsoft Excel 2011 Office system. The variables included were summarized according to their type. For the statistical analysis of the results, the SPSS system was used (version 11.5, SPSS Inc., New York) and thus the determination of the different estimators. Descriptive measures were used to summarize the data. Absolute and relative frequencies expressed in percentages were used for the qualitative variables. For the quantitative variables, descriptive statistics, averages and standard deviation were implemented.

\section{Results}

Fifty patients with a diagnosis of gigantomastia, treated with reductive mastoplasty with a lower pedicle flap and free NAC graft were studied. The group of women aged less than 50 years predominated with $76 \%$ and the average age was 41.4 years. Extreme ages ranged from 19 to 66 years with a standard deviation of \pm 12.7 years. Obesity and overweight were predominant variables in this type of patient. The mean body mass index obtained was 31.64 , which shows the relationship between obesity and gigantomastia.

The average preoperative breast volume was $1858.60 \mathrm{gr}$. for the left breast and $1852.40 \mathrm{gr}$. for the right, whose limits ranged from 1500 to 4500 gr. with a standard deviation of 508.65 and 504.39 gr. respectively. This demonstrates further development of the left breast.

The average of resected tissue was 1142.80 gr. for the left breast and 137.60 gr. for the right, (Figure 3) with standard deviation of $448.4 \mathrm{gr}$. in the first and 436.9 gr. for the second. (Table 1) The average posoperative volume recorded was 706.80 gr., with extreme values of 1000 and 500 gr. and standard deviation of $102.72 \mathrm{gr}$.

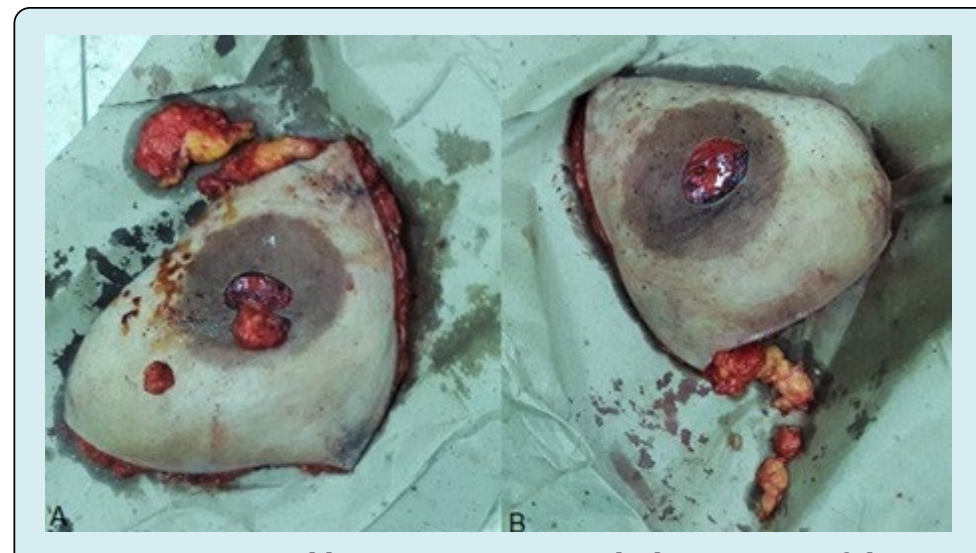

Figure 3: Resected breast tissue. A: Right breast. B: Left breast. 


\begin{tabular}{|c|c|c|c|c|}
\hline Resected breast tissue & Maximum (grams) & Mínimum (grams) & Average & Standard Deviation \\
\hline Rigth Breast & 3500 & 700 & 1137.60 & 436.9 \\
\hline Left Breast & 3350 & 800 & 1142.80 & 448.4 \\
\hline
\end{tabular}

Table 1: Volume of resected breast tissue.

Source: Clinical Histories.

There were no intraoperative anesthetic complications related to the procedure. The $4 \%$ of the patients presented acute anemia related to resections of large tissue volumes that required blood volume control, plasma expanders, and blood products.

There were $8 \%$ of the patients presented dehiscence of the surgical wound at the junction of the vertical and horizontal closure, less than $2 \mathrm{~cm}$ in diameter and closed by second intention. Of these, $4 \%$ evolved satisfactorily and the other $4 \%$ developed unsightly scars. Only 1 patient (2\%) developed a widened scar in the horizontal incision in the late postoperative period that improved at the end of healing in the remodeling phase. The other $2 \%$ presented hypertrophic scarring in the submammary sulcus and a keloid scarring in the late posoperative period that improved with treatment with silicone sheets and direct compression. In none of the cases was surgical treatment required to correct complications.

The evolution of the results was $92 \%$ good, $8 \%$ fair and in none of the cases was it bad.

The patients were fully satisfied (Figures $4 \& 5$ ).

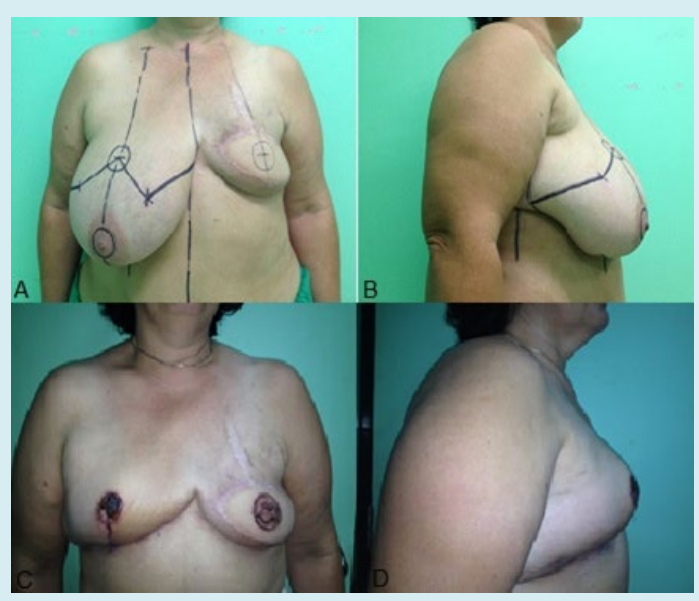

Figure 4: Oncological deformity of the breast. A: Preoperative - right gigantomastia with posoperative left asymmetry - front view. B: Preoperative - right lateral view. C: Posoperative at 21 days- frontal view. D: Posoperative at 21 days- right lateral view.

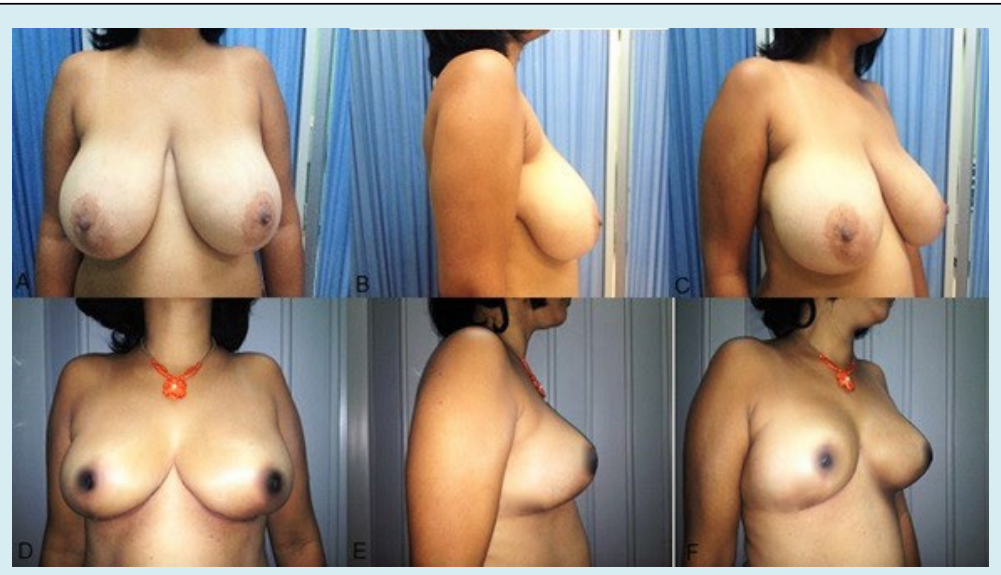

Figure 5: Gigantomastia plus severe ptosis. A: Preoperative - front view. B: Preoperative- profile view. C: Preoperative- oblique view. D: Posoperative at 6 months- frontal view. E: Posoperative at 6 months - profile view. F: Posoperative at 6 months oblique view. 


\section{International Journal of Transplantation \& Plastic Surgery}

\section{Discussion}

The predominant age group in the 41-year-old study is related to the period of the woman who usually presents physical changes due to age, lifestyles, and satisfied parity, and therefore probably requires this type of procedure. Manuela Berrocal in her book The Four Seasons of Breast attributes the progressive increase of the breasts to genetic, hereditary, ethnic, hormonal, nutritional factors or associated diseases such as obesity [19]. However, breast reduction surgery, like all surgical procedures. It has risks and complications, which can be common to the rest of the surgical interventions or specific to the technique.

The international literature is diverse with respect to age groups, but the majority agrees with this study at the ages of greatest request for this procedure. Vicente registers an average of 38 years with extreme ages between 15 and 56 years [20]. Yepez Intriago in his article on reduction mammoplasty with small scars for large breast hypertrophies or gigantomastia, describes patients with ages between 16 and 70 years, the group that most requested this procedure between the ages of 20 and 30 [21]. Agbernorku used the NAC free grafting technique in gigantomastia in a group of patients whose ages ranged from 12 to 28 years, with a mean of 16 years [22], which differs from this series. Sanchez Castillo published an article on minimal incisions for reducing mastoplasties, where ages between 30 and 39 predominated [23].

The maximum breast volume recorded in the preoperative period was 4500 gr. and the black race predominated. Grippaudo, et al. in a 2009 study on breast reduction by liposuction method only, observed that the cases studied showed greater growth of the left breast [24], which coincides with this study, which had an average of 1858.60 gr. for the left breast, which shows greater development of the left breast compared to the right.

In all the operated patients, breast symmetry was achieved, with a conical shape and adequate projection in harmony with its biotype and characteristics of the thoracic region. The posoperative measurements were within the parameters established in an ideal breast. The regular results were due to complications that affected good healing.

The average resected breast volume was 1140.20 gr., with a variation from 700 to 3500 gr. By reducing large volumes of breast tissue, sizes can be obtained with a final volume of up to $1000 \mathrm{gr}$. The technique used allows predetermining the amount of tissue to be resected from planning. In the posoperative period the average breast volume was $706.80 \mathrm{gr}$ which, according to the Roa classification, are patients with light, moderate and marked hypertrophies. He considers that the ideal breast pattern varies according to biotype, height and weight. According to these parameters, the breast reduction must be done to obtain a result that is consistent with the body harmony of the patients [17].

The complications reported in the study are few, the majority being partial suture dehiscence, followed by acute anemia, which required medical treatment. However, these only partially coincide with the most frequent complications reported in the international literature for reductive mastoplasty techniques in large breast hypertrophies that vary from different necrosis (total or partial NAC, total or partial flap, steatonecrosis) to seromas, bruises and dehiscence of the sutures.

In the present study, the main complication was suture dehiscence $<2 \mathrm{~cm}$ at the junction of the vertical inverted $\mathrm{T}$ closure with the horizontal closure by second intention. Similar results are reported by Mandrekas et al. in 371 reductive mastoplasties with lower pedicle with resection of large volumes of tissue, (870 gr. as average of tissue removed by each breast) with $11.4 \%$ of complications and $4.6 \%$ were dehiscence of the wound at the $\mathrm{T}$ junction with closure by ulterior motive [25].

Patients are presented in the series with alterations in hypertrophic scar-like healing at different times of the healing process and in the late posoperative period. This coincides with Ahmed and Kolhe's comparative study of reductive posmastoplasty areolar sensitivity between the inferior pedicle flap technique and free graft of the NAC, whose most frequent complication was 3.3\% hypertrophic scar, followed by fatty necrosis and necrosis of the NAC with the same frequency $0.8 \%[26]$.

This study reports good aesthetic results, satisfaction of all patients and low incidence of complications, similar to the literature consulted. There is similarity in the volume of resected tissue, the versatility is greater when breast tissue is resected using the reductive mastoplasty technique with inferior pedicle flap and free NAC graft.

With this surgical technique, total patient satisfaction is achieved. This could be verified because there was remission of the symptoms in general, regional and local (respiratory restriction, radiculitis, grooves in the shoulders, intertrigo and cervicothoracic pain). They reintegrated into social, work and couple life. Currently, they can find clothing suitable for their age and biotype, which reinforces their self-esteem and self-image, a binomial whose integrity is synonymous with 


\section{International Journal of Transplantation \& Plastic Surgery}

health and quality of life.

\section{Conclusions}

Reductive mastoplasty with an inferior pedicle flap and free grafting of the areola-nipple complex is a surgical treatment option for gigantomastia that offers good results, few complications and high patient satisfaction.

\section{References}

1. Pitanguy I, Radwanski HN (2016) Correction of breast hypertrophies and hypomastia In: Coiffman F (Ed.) Coiffman reconstructive and aesthetic plastic surgery. $4^{\text {th }}$ (Edn.), Bogota Amolca pp: 1857-1868.

2. Jimenez Reyes W, Rodriguez Gil E (2018) Reductive mastoplasty by modified Thorek technique in gigantomastia. Rev Inform Cient 97(1): 183-191.

3. Cerqueira A (1998) Mammoplasty: breast fixation with dermoglandular mono upper pedicle flap under the pectoralis muscle. Aesthet Plast Surg 22(4): 276-283.

4. Alviar Rueda JD (2018) Benign breast pathology: diagnosis and treatment. Med Uis 31(3): 61-71.

5. Palmuth $\mathrm{T}$ (1648) Observations medicuarumcentinae trees posthumae. Braunschweig: Springer-Verlag.

6. Dafydd H, Roehl KR, Phillips LG, Dancey A, Peart F, et al. (2011) Redefining gigantomastia. J Plast Reconstr Aesthet Surg 64(2): 160-163.

7. Chiu TW (2011) Stone's plastic surgery facts and figures. $3^{\text {rd }}$ (Edn.) Cambridge University Press New York.

8. Fonseca CC, Veiga DF, Garcia EDS, Cabral IV, de Carvalho $M M$, et al. (2018) Breast Hypertrophy, Reduction Mammaplasty, and Body Image. Aesthet Surg J 38(9): 972-979.

9. Perez Panzano E, Gascon Catalan A, Sousa Dominguez R, Carrera Lasfuentes P, Garcia Campayo J, et al. (2017) Reduction mammaplasty improves levels of anxiety, depression and body image satisfaction in patients with symptomatic macromastia in the short and long term. J Psychosom Obstet Gynaecol 38(4): 268-275.

10. Sharma K, Nigam S, Khurana N, Chaturvedi KU (2004) Unilateral gestational macromastia--a rare disorder. Malays J Pathol 26(2): 125-128.

11. Charles H. Thorne (2014) Spear S Breast reduction: inverted-t. In: Barcelona S (Eds.), Grabb and Smith's Plastic Surgery. 7th (Edn.), pp: 1157-1159.
12. Latrenta G, Hoffman L, Rees T, Latrenta G (1994) Breast reductions In: Aesthetic plastic surgery. Saunders Company, Philadelphia, pp: 927-932.

13. Wise RJ (1946) preliminary report on a method of planning the mammoplasty. Plast Reconstr Surg. 17(5): 367-375.

14. Regnault P (1980) Breast reduction: B- Technique. Plast Reconstr Surg 65(6): 840-845.

15. Courtis E, Goldwyn R (1997) Reduction mammaplasty by the inferior pedicle technique. An alternative to free nipple and areola grafting for severe macromastia or extreme ptosis. Plast Reconstr Surg 59(4): 500-507.

16. Robbins TH (1997) A reduction mammoplasty with the areole-nipple based on an inferior pedicle. Plast Reconstr Surg 59(1): 64-67.

17. Dieppa Ramires OA, Alba Matos E (2013) Impact of reductive mastoplasty with minimal incision techniques. 65: 15 .

18. Bonilla-Sepulveda OA (2016) Virginal mammary hyperplasia associated with diffuse mammary stromal pseudoangiomatous hyperplasia. Rev CES Med 30(1): 122-128.

19. Berrocal Revueltas M (2014) The four seasons of the breasts. $1^{\text {st }}(E d n$.$) , Bogota, pp: 25-26.$

20. Peña Vicente S, Gonzalez Peirona E, Guemes Sanchez A, Sousa Dominguez R, Garcia-Campayo J (2003) Reduction mammoplasty: surgery for a psychosocial problem. Senol Patol Mamaria 16: 53-57.

21. Yepez Intriago M, Estrella Tejada P, Velez Polit E (2013) Reduction mammoplasty with small scars for large breast hypertrophies or gigantomastia. Cir Plast Iberolatinoam 39: 1-8.

22. Agbenorku P (2006) Nipple-Areolar Complex (NAC) composite grafts in the management of macromastia: review of complications. Nigerian J Surg Res 8(1): 86-89.

23. Sanchez Castillo VM, Collado Hernandez CM (2002) Minimal incisions for reducing mastoplasty. Rev Cub Cir 41: 11-15.

24. Grippaudo FR, Kennedy DC, Tiwari P, Talavera F, Shenaq SM, et al. (2015) Liposuction only breast Reduction. Medscape Plastic Surgery \& Aesthetic Medicine.

25. Mandrekas A, Zambacos GJ (1996) Reduction mammaplasty with the inferior pedicle technique: early and late complications in 371 patients. Brit J Plast Surg 


\section{International Journal of Transplantation \& Plastic Surgery}

49: $442-446$.

26. Ahmed OA, Kolhe PS (2000) Comparison of nipple and areolar sensation after breast reduction by free nipple graft and inferior pedicle techniques. Br J Plast Surg 53(2): 126-129.

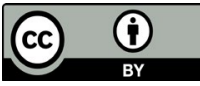

